\title{
LYAPUNOV EXPONENTS FOR DISCONTINUOUS DIFFERENTIAL EQUATIONS
}

BY

\author{
B. S. BERGER AND M. ROKNI
}

University of Maryland, College Park, Maryland

\begin{abstract}
The vector field associated with a dynamical system is assumed to be piecewise continuously differentiable. The gradient of the vector field, entering into integral expressions for the Lyapunov exponents, may therefore contain derivatives of step functions. Results from the theory of distributions are used in the integrals' evaluation.
\end{abstract}

Introduction. Differential equations have been previously derived which describe the evolution of vectors, area tensors, and Lyapunov exponents along a trajectory. These equations have been integrated by various means to give the spectrum of Lyapunov exponents, L. E., $[6,10]$. For discontinuous differential equations, D. D. E., $\exists$ a set of points, $c \in \mathbb{R}$, for which the integrands of the evolution equations, gradients of the vector field, will contain distributions. It is assumed that each point of the set $c_{i} \in\left(a_{i}, b_{i}\right)$, where the intervals are disjoint. Integrals for the evolution of vectors, phase space volume, and $\mathrm{L}$. E. are evaluated over the intervals $\left[a_{i}, b_{i}\right]$. The integration methods of $[6,10]$ are applicable to the complement of $\left(a_{i}, b_{i}\right)$.

D.D.E. occur in a variety of applications including relay control systems $[2,3]$ and the oscillation of mechanical systems with Coulomb friction. Such systems may exhibit intermittent motion. A numerical study indicates the utility of the derived algorithms.

Lyapunov exponents. Consider a dynamical system

$$
\dot{\mathbf{x}}=\mathbf{f}(\mathbf{x})
$$

where $\mathbf{x}$ are spatial curvilinear coordinates in an $n$-dimensional Euclidean phase space, $E_{n}$, and $\mathbf{f}(\mathbf{x})$ is piecewise continuously differentiable. Under general conditions the solution of (1),

$$
\mathbf{x}=\mathbf{x}(\mathbf{X}),
$$

exists, is unique and continuous, [1,3], where $\mathbf{X}$ are material coordinates.

A subspace, $V_{m}$, of dimension $m \leq n$ may be defined by the parametric equations $\mathbf{X}=\mathbf{X}(\mathbf{u})$ in material coordinates and after deformation, (2), by $\mathbf{x}=\mathbf{x}(\mathbf{u})$ in spatial coordinates, where $\mathbf{u}$ has $m$ components. Let $d a_{(m)}$ and $d A_{(m)}$ be spatial and 
material area elements of $V_{m}$, respectively. The $m$-dimensional L.E. associated with a trajectory originating at $\mathbf{X}$ is defined as, [4,5],

$$
\chi\left(\mathbf{X}, V_{m}\right) \equiv \limsup _{t \rightarrow \infty}\left(\ln \left(d a_{m} / d A_{m}\right)\right) / q(t)
$$

where $q(t) \equiv t$. The one-dimensional L.E., $m=1$, for the arc, $d s$, with tangent, $\mathbf{n}$, associated with a trajectory and having initial values, respectively, of $d S, \mathbf{N}$, and $\mathbf{X}$ is given by

$$
\chi(\mathbf{X}, d \mathbf{s})=\lim _{t \rightarrow \infty}\left(\ln \lambda_{\mathbf{n}}\right) / q(t)
$$

where stretch $\lambda_{\mathbf{n}} \equiv d s / d S$. Since $D\left(\ln \lambda_{\mathbf{n}}\right) / D t=d_{k 1} n_{k} n_{1},|\mathbf{n}|=1$, it follows that (4) may be expressed as

$$
\chi(\mathbf{X}, d \mathbf{s})=\lim _{t \rightarrow \infty}(1 / q(t)) \int_{0}^{t} d_{k 1} n_{k} n_{1} d t
$$

where $d_{k 1} \equiv\left(v_{k, 1}+v_{1, k}\right) / 2$ and $v_{k} \equiv \dot{x}_{k}$.

The material derivative of a vector, $\mathbf{n}$, tangent to an arc $d s$ is given by, [6,7], $D\left(n_{k}\right) / D t=v_{k, 1} n_{1}$ and therefore

$$
n_{k}(t)=n_{k}(0)+\int_{0}^{t} v_{k, 1} n_{1} d t
$$

In $[4,5,6]$ an algorithm is given for the computation of $\chi_{(\alpha)}\left(\mathbf{X}, d \mathbf{s}_{\alpha}\right), \alpha=1, \ldots$, $n$. An arbitrary set of initial orthogonal unit vectors, $\left\{\mathbf{n}_{1}, \ldots, \mathbf{n}_{n}\right\}$, is evolved through the numerical solution of (1) and (6). At each time step the vectors are orthonormalized. $\chi_{(\alpha)}\left(\mathbf{X}, d \mathbf{s}_{\alpha}\right)$ is then given by (5). In [4,6] it is shown that $\sum \chi_{i}\left(\mathbf{X}, d \mathbf{s}_{i}\right)=\chi\left(\mathbf{X}, V_{m}\right), i=1, m$, and that

$$
\chi\left(\mathbf{X}, V_{n}\right)=\lim _{t \rightarrow \infty}(1 / q(t)) \int_{0}^{t} v_{k, k} d t .
$$

D.D.E. Since $\mathbf{f}(\mathbf{x})$ in (1) is piecewise continuously differentiable, the gradient, $v_{i, j}$ may contain derivatives of step functions. The following results are useful in evaluating the integrals of such $v_{i, j}$ as they occur in $(5,6,7),[8,9]$ : If, in some neighborhood of $t=c, g(t)$ is continuous, $x(t)$ is continuous and monotonic, $x(c)=0$, and $\dot{x}\left(c^{+}\right) \exists$ and does not vanish then

$$
\int_{0}^{t} g(t) \frac{\partial u(x(t))}{\partial x} d t=g(c) /\left|\dot{x}\left(c^{+}\right)\right|
$$

where $u(x)=1$ for $x>0, u(x)=0$ for $x<0$, and $0<c<t$. Since $\operatorname{sgn}(x)=$ $u(x)-u(-x)$ it follows from $(8)$ that

$$
\int_{0}^{t} g(t) \frac{\partial \operatorname{sgn}(x(t))}{\partial x} d t=g(c)\left(1 /\left|\dot{x}\left(c^{-}\right)\right|+1 /\left|\dot{x}\left(c^{+}\right)\right|\right) .
$$

Asymmetric relay elements and relays with hystersis and dead zones may be modeled by sums of $u(x)$ and $\operatorname{sgn}(x)$, [2]. The function describing the behavior of a relay with a dead zone is given by $\left(\operatorname{sgn}\left(x-x_{0}\right)+\operatorname{sgn}\left(x+x_{0}\right)\right) / 2$ which $=1,0,-1$ for $x>x_{0},|x|<x_{0}$, and $x<-x_{0}$, respectively. 
An example will indicate the general application of (8) and (9). Consider an oscillator with Coulomb damping:

$$
\begin{aligned}
& \dot{x}_{1}=x_{2} \\
& \dot{x}_{2}=-k_{1} x_{1}-F \operatorname{sgn}\left(x_{2}\right)+f\left(x_{3}\right) \\
& \dot{x}_{3}=1
\end{aligned}
$$

where the constant coefficients, $k_{1}$ and $F$, are $\geq 0$. Then $v_{1,1}=0, v_{1,2}=1$, $v_{1,3}=0, v_{2,1}=-k_{1}, \quad v_{2,2}=-F \partial \operatorname{sgn}\left(x_{2}\right) / \partial x_{2}, \quad v_{2,3}=\partial f / \partial x_{3}, \quad v_{3,1}=0$, $v_{3,2}=0$, and $v_{3,3}=0$. (7) and (9) imply that

$$
\chi\left(\mathbf{X}, V_{n}\right)=\lim _{t \rightarrow \infty}(-F / t) \sum_{i}\left(1 /\left|\dot{x}_{2}\left(c_{i}^{-}\right)\right|+1 /\left|\dot{x}_{2}\left(c_{i}^{+}\right)\right|\right)
$$

where $x_{2}\left(c_{i}\right)=0$. In an interval, $a \leq t \leq b$, with $k=1$, (6) implies

$$
n_{1}(b)-(h / 2) n_{2}(b) \simeq n_{1}(a)-(h / 2) n_{2}(a)
$$

where $h \equiv b-a$ and the trapezoidal integration approximation has been applied. For $k=3$ it follows from (6) that $n_{3}(b)=n_{3}(a)$. Since the initial values of $n_{i}$ are arbitrary, let $n_{3}=0$ for $0 \leq t$. It then follows from (6) with $k=2$ that

$$
\left(k_{1} h / 2\right) n_{1}(b)+\left(1+F /\left|\dot{x}_{2}(b)\right|\right) n_{2}(b) \simeq-\left(k_{1} h / 2\right) n_{1}(a)+\left(1-F /\left|\dot{x}_{2}(a)\right|\right) n_{2}(a)
$$

where $a<c<b, \dot{x}_{2}\left(c^{-}\right) \simeq \dot{x}_{2}(a)$, and $\dot{x}_{2}\left(c^{+}\right) \simeq \dot{x}_{2}(b)$. (14) and (15) determine $\mathbf{n}(b), A \mathbf{n}=\mathbf{d}$, and $\operatorname{det}|A|>1$.

Integration of $D\left(\ln \lambda_{\mathrm{n}}\right) / D t,(4)$, and (5) for $a \leq t \leq b$ gives

$$
\begin{aligned}
\ln \lambda_{\mathbf{n}}(b)= & \ln \lambda_{\mathbf{n}}(a)+\left(h\left(1-k_{1}\right) / 2\right)\left(n_{1}(b) n_{2}(b)+n_{1}(a) n_{2}(a)\right) \\
& -F\left(n_{2}^{2}(b) /\left|\dot{x}_{2}(b)\right|+n_{2}^{2}(a) /\left|\dot{x}_{2}(a)\right|\right) .
\end{aligned}
$$

If $h \ll 1$ and $k_{1} h \ll 1$ then terms involving products with $h$ may be omitted in (14), (15), and (16).

Solutions of D.D.E. may be intermittent in the sense that for some time interval, $\alpha<t<\beta$, components of $\dot{\mathbf{x}}=0$. If the system (10), (11), and (12) stops at $t=\alpha$ then $\dot{x}_{1}(\alpha)=0, \dot{x}_{2}\left(\alpha^{-}\right) \neq 0, \dot{x}_{2}\left(\alpha^{+}\right)=0$, and $x_{3}(t) \neq 0$. In (9) the term containing $\left|\dot{x}\left(c^{+}\right)\right|$is therefore omitted.

Computation of L.E. The oscillator with Coulomb damping is subsequently used as a test case. Equations (10), (11), and (12) were solved simultaneously assuming $f\left(x_{3}\right) \equiv 0, F=5.886, k_{1}=60, x_{1}=40$, and $x_{2}=0$ at $x_{3}=0 . \quad \chi\left(\mathbf{X}, V_{2}\right)$, (7), was evolved through (13) and is shown in Fig. 2 for $0 \leq t \leq 50$. Two vectors, $\mathbf{n}^{1}$ and $\mathbf{n}^{2}$, were evolved with $(6)$ and in intervals containing points $c_{i}$ by (14) and (15). These were orthonormalized after each time step. The evolution of $\chi_{1}$ and $\chi_{2}$ associated with $\mathbf{n}^{1}$ and $\mathbf{n}^{2}$ was realized through (5) and (16). $\chi_{1}$ and $\chi_{2}$ are shown in Fig. 1 for $0 \leq t \leq 50$. The numerical error associated with the equality $\chi\left(\mathbf{X}, V_{2}\right)=\chi_{1}\left(\mathbf{X}, d \mathbf{s}^{1}\right)+\chi_{2}\left(\mathbf{X}, d \mathbf{s}^{2}\right)$ was $<1 \%$. Since this system stops, it follows that 0 is the asymptotic limit of $\chi_{1}, \chi_{2}$, and $\chi$ which is approached as $1 / t$. 


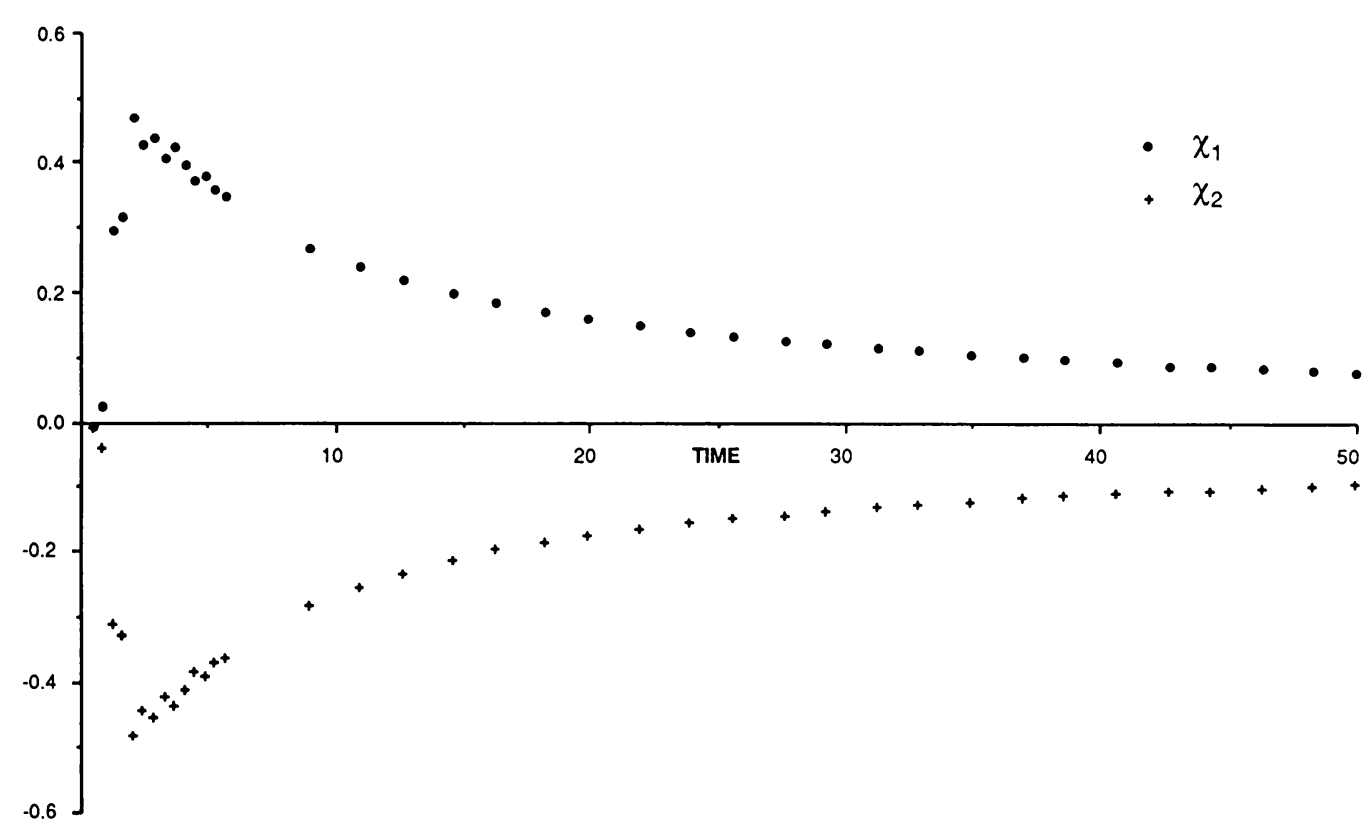

FIG. 1 .

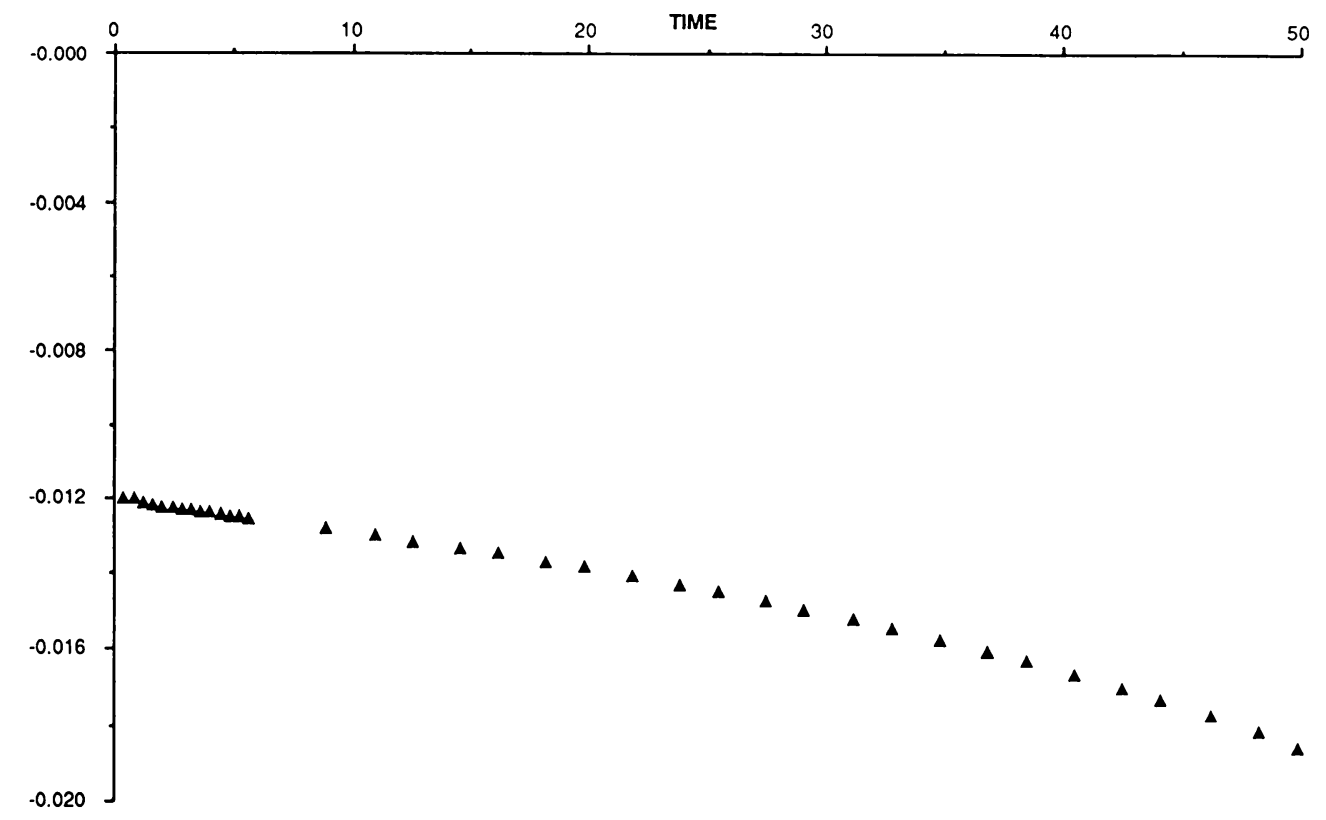

FIG. 2.

Conclusions. Integral expressions for L.E. appear to be particularly appropriate for vector fields with gradients containing distributions. An approximation of (7) was studied for (10), (11), and (12), in which $\operatorname{sgn}(\dot{x})$ was approximated by $a(\dot{x})=+1$, 
$\dot{x}>\varepsilon, a(\dot{x})=-1, \dot{x}<\varepsilon$, and $a(\dot{x})=\varepsilon \dot{x}$ for $|\dot{x}| \leq 1$. The results were in very close agreement with those of (13) for small $\varepsilon$.

Acknowledgment. The authors gratefully acknowledge the assistance of C. J. Berger and the encouragement of Dr. Matthew Gordon-Clark. Computations were supported by the Computer Science Center of the University of Maryland.

\section{REFERENCES}

[1] J. Guckenheimer and P. Holmes, Nonlinear Oscillations, Dynamical Systems and Bifurcations of Vector Fields, Springer-Verlag, 1983

[2] Y. Z. Tsypkin, Relay Control Systems, Cambridge University Press, 1984

[3] A. A. Andronov, E. A. Vitt, and S. E. Khaiken, Theory of Oscillators, Pergammon Press, 1966

[4] G. Benettin, L. Galagani, A. Giorgilli, and J. Strelcyn, Lyapunov characteristic exponents for smooth dynamical systems and for Hamiltonian systems: method for computing all of them, Meccanica 15, 9-20 (1980)

[5] J. Shimada and T. Nagashima, A numerical approach to the ergodic problem of dissipative dynamical systems, Prog. in Theoretical Physics (6) 61, 1605-1616 (1979)

[6] B. S. Berger and M. Rokni, Lyapunov exponents and continuum kinematics, Int. J. Engrg. Sci. 25, 1079-1084 (1987)

[7] A. C. Eringen, Continuum Physics, vol. II, Academic Press, 1975

[8] A. H. Zemanian, Distribution Theory and Transform Analysis, McGraw-Hill, 1965

[9] D. H. Griffel, Applied Functional Analysis, Ellis Horwood Limited, 1981

[10] M. Rokni and B. S. Berger, Lyapunov exponents and subspace evolution, Quart. Appl. Math. (4) 45, 789-793 (1987) 